\title{
Bronchial Obstruction, CTCAE
}

National Cancer Institute

\section{Source}

National Cancer Institute. Bronchial Obstruction, CT CAE. NCI Thesaurus. Code C143340.

A disorder characterized by blockage of a bronchus passage, most often by bronchial secretions and exudates. 